\title{
Using spectral methods to obtain particle size information from optical data: applications to measurements from CARES 2010
}

D. B. Atkinson et al.

Correspondence to: Dean B. Atkinson (atkinsond@pdx.edu)

The copyright of individual parts of the supplement might differ from the CC BY 4.0 License. 


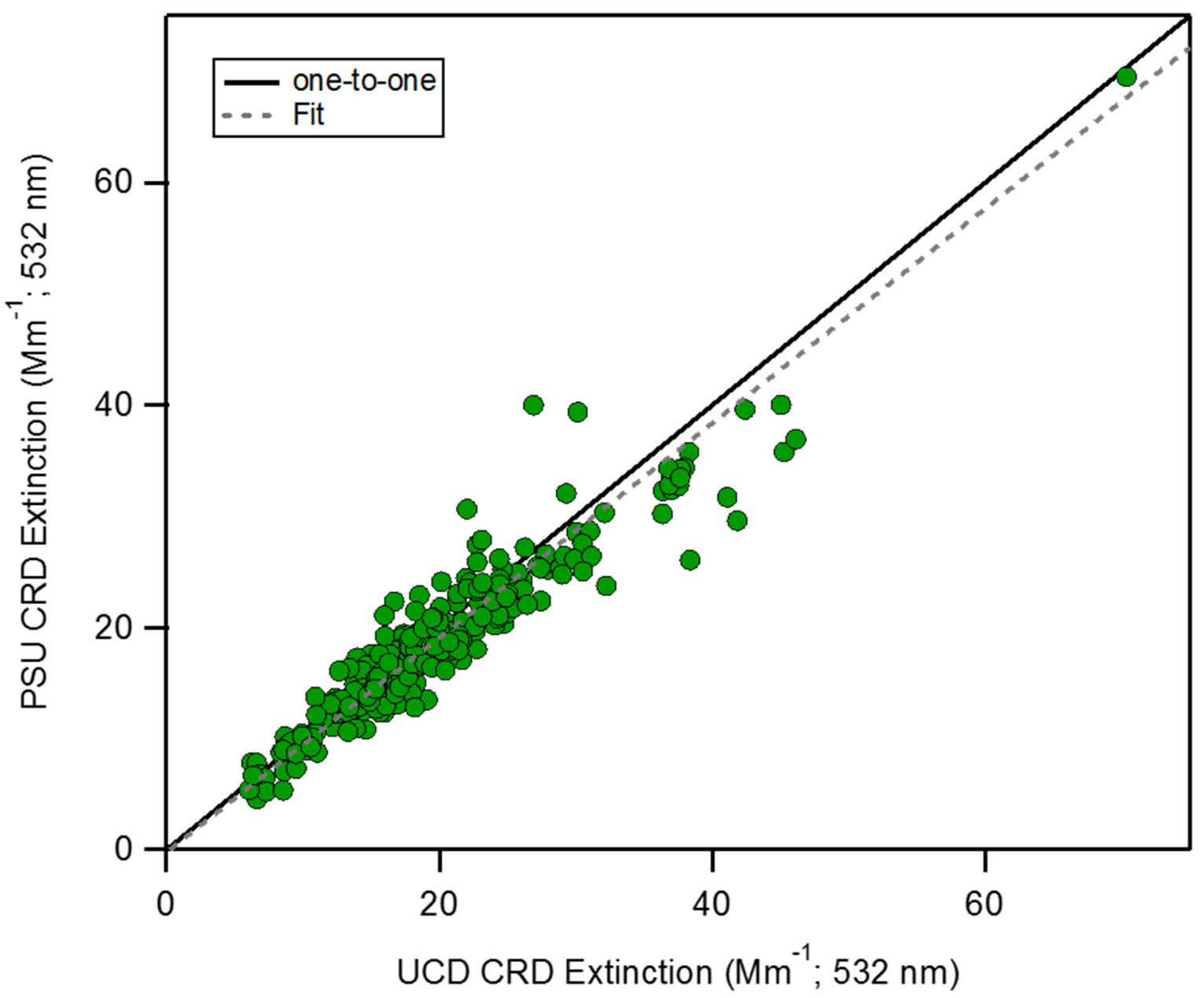

Figure S1. Scatterplot of the visible $(532 \mathrm{~nm})$ extinction measurements from the two CRD instruments used at TO. The solid line in the figure is the 1:1 line while the dashed is the result of an orthogonal distance regression that produced a slope of 0.96 and a statistically insignificant intercept. Units on both axes are $\mathrm{Mm}^{-1}$.

26 

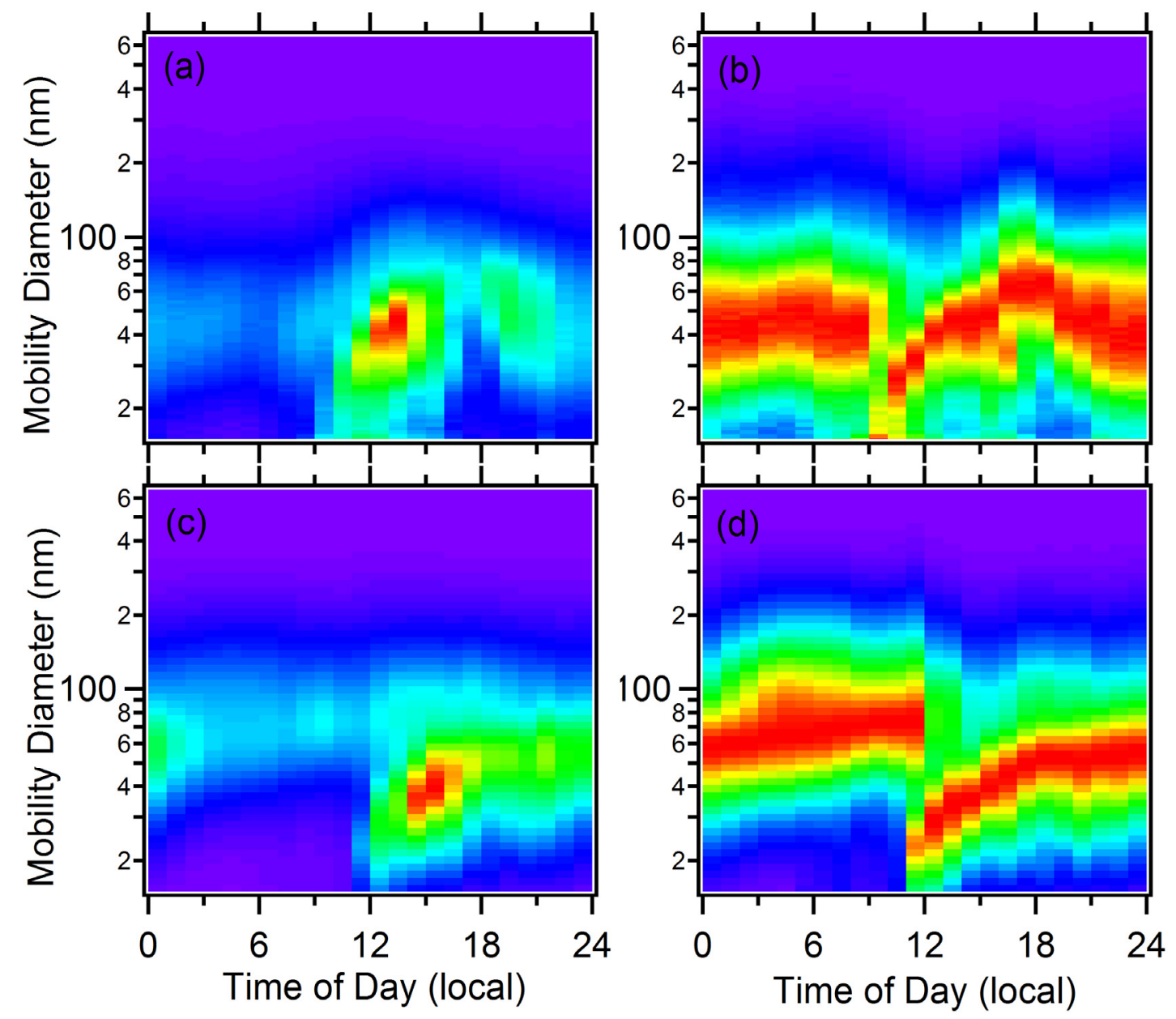

Figure S2. Observed diurnal variability in the number-weighted mobility size distribution $\left(d N / d \log D_{p}\right)$ for $(a, b)$ T0 and (c,d) T1. The color corresponds to particle concentration. $(a, c)$ The unnormalized data, with the red indicating the period with the highest concentration. $(b, d)$ The size distribution where each hour average is normalized to the maximum concentration during that hour. The appearance of a mode associated with new particle formation and growth starting at 8 am at T0 and at 11 am at T1 is evident. Data were averaged for June 21-29, 2010. 

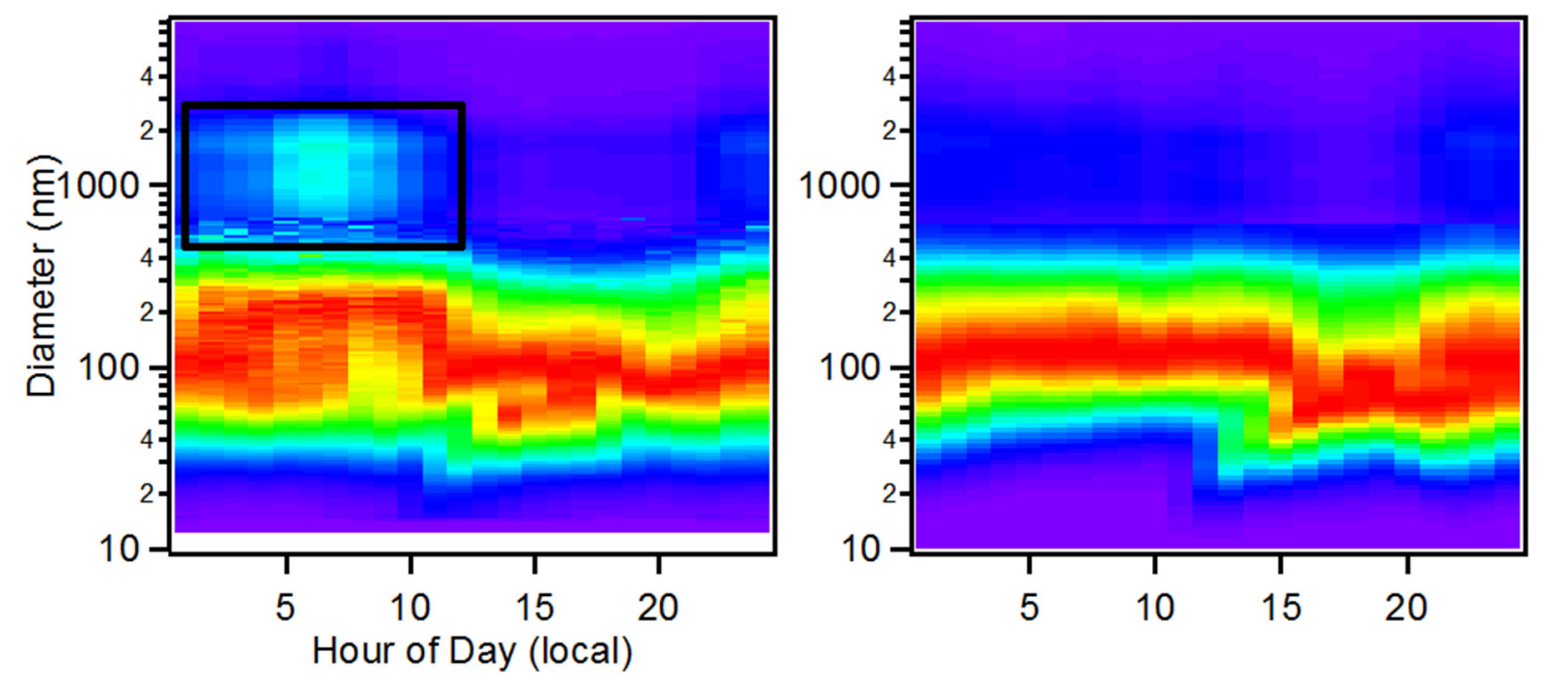

36

Figure S3. Observed diurnal variation for (left) the T0 site and (right) the T1 site for the surfacearea weighted size distribution. Distributions have been normalized to the maximum surface area concentration for each hour of the day. The black box shown for T0 highlights the presence of a mode near 1 micron.

40 